\title{
Highly Strained III-V-V Coaxial Nanowire Quantum Wells with Strong Carrier Confinement
}

Yunyan Zhang, ${ }^{*}{ }^{\dagger}$ George Davis, ${ }^{\dagger}$ H. Aruni Fonseka, ${ }^{\# \odot ~ A n t o n ~ V e l i c h k o, ~}{ }^{\ddagger}$ Anders Gustafsson, ${ }^{\S}{ }^{\circledR}$ Tillmann Godde, ${ }^{\ddagger}$ Dhruv Saxena, ${ }^{4}$ Martin Aagesen, $"$ Patrick W. Parkinson, ${ }^{\perp} \odot$ James A. Gott, Suguo Huo, ${ }^{\nabla}$ Ana M. Sanchez, ${ }^{\#[0}$ David J. Mowbray, ${ }^{\ddagger}{ }^{\circ}$ and Huiyun Liu ${ }^{\dagger}$

${ }^{\dagger}$ Department of Electronic and Electrical Engineering, University College London, London WC1E 7JE, United Kingdom

${ }^{\ddagger}$ Department of Physics and Astronomy and the Photon Science Institute, University of Sheffield, Sheffield S3 7RH, United Kingdom

\#Department of Physics, University of Warwick, Coventry CV4 7AL, United Kingdom

${ }^{\S}$ Solid State Physics and NanoLund, Lund University, Box 118, SE-221 00 Lund, Sweden

${ }^{I}$ The Blackett Laboratory, Department of Physics, Imperial College London, London SW7 2AZ, United Kingdom

"Danish Defence Research Center, Lautrupbjerg 1-5, 2750 Ballerup, Denmark

${ }^{\perp}$ School of Physics and Astronomy and the Photon Science Institute, University of Manchester, Manchester M13 9PL, United Kingdom

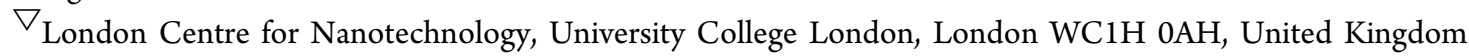

Supporting Information
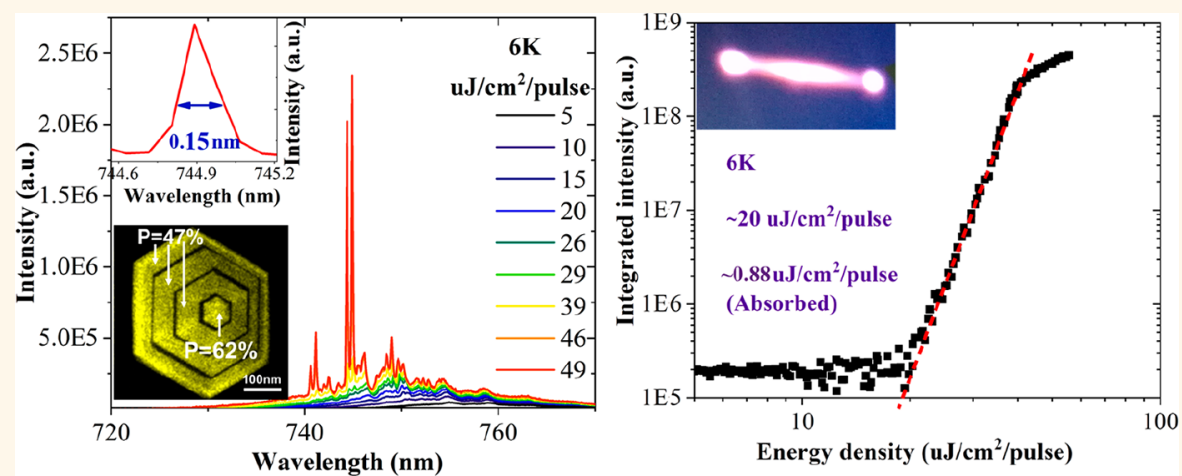

ABSTRACT: Coaxial quantum wells (QWs) are ideal candidates for nanowire (NW) lasers, providing strong carrier confinement and allowing close matching of the cavity mode and gain medium. We report a detailed structural and optical study and the observation of lasing for a mixed group-V GaAsP NW with GaAs QWs. This system offers a number of potential advantages in comparison to previously studied common group-V structures (e.g., AlGaAs/GaAs) including highly strained binary GaAs QWs, the absence of a lower band gap core region, and deep carrier potential wells. Despite the large lattice mismatch $(\sim 1.7 \%)$, it is possible to grow defect-free GaAs coaxial QWs with high optical quality. The large band gap difference results in strong carrier confinement, and the ability to apply a high degree of compressive strain to the GaAs QWs is also expected to be beneficial for laser performance. For a non-fully optimized structure containing three QWs, we achieve low-temperature lasing with a low external (internal) threshold of $20(0.9) \mu \mathrm{J} / \mathrm{cm}^{2} / \mathrm{pulse}$. In addition, a very narrow lasing line width of $\sim 0.15 \mathrm{~nm}$ is observed. These results extend the NW laser structure to coaxial III-V-V QWs, which are highly suitable as the platform for NW emitters.

KEYWORDS: nanowire, III-V-V, quantum well, carrier collection, carrier confinement, laser

emiconductor nanowires (NW) lasers provide strong optical mode confinement in a very small volume, allowing laser diameters on the order of a few hundred
Received: March 5, 2019

Accepted: May 8, 2019

Published: May 8, 2019 


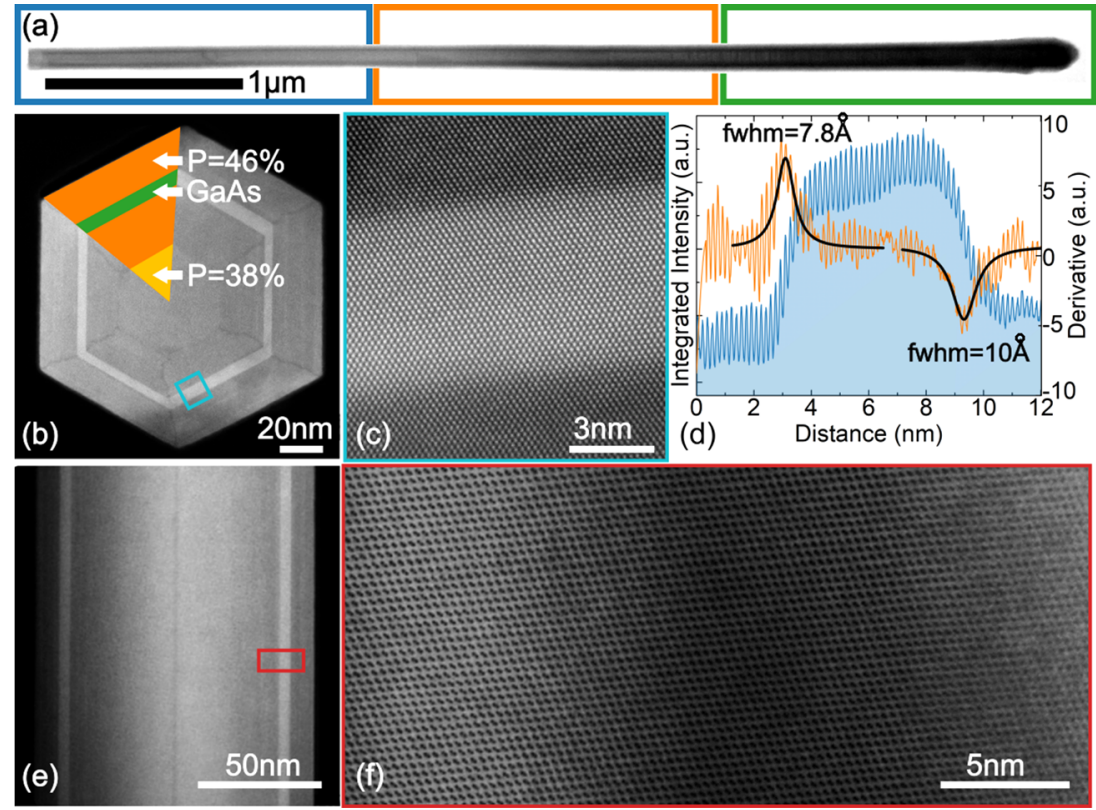

Figure 1. Crystalline properties of GaAsP nanowires with a single coaxial GaAs QW. (a) Low-magnification bright field scanning transmission electron microscopy (BF-STEM) image showing one entire NW. The blue, yellow, and green rectangles indicate the twin-free, slightly twinned, and defect-containing regions, respectively. (b) Annular dark field STEM (ADF-STEM) image showing a cross-section from the stacking-fault-free end of the NW. The white region is the GaAs QW. The inset is a schematic of the structure. (c) Atomic-resolution ADF-STEM image of the QW region. (d) Energy dispersive X-ray spectroscopy (EDX) line profile of the As concentration across the QW. The sharpness of the two interfaces is more easily seen from the first derivative of the As concentration, the orange data with the black line being a fit. (e) Low-resolution STEM image from the stacking-fault-free section of the NW, imaged along the $\langle 112\rangle$ zone axis. The two pale lines represent the GaAs QW. (f) Atomic-resolution STEM image of the region delimited by the red box in (e).

nanometers and length of a few micrometers. ${ }^{1-3}$ They hence provide small-size, low-power devices with a wide range of applications. Moreover, III-V NW structures can be grown on a $\mathrm{Si}$ platform, ${ }^{4-6}$ thus enabling fabrication of high-quality photonic light sources for low-cost, ultra-high-density integration, solving one of the major challenges currently limiting the exploitation of $\mathrm{Si}$ photonics. ${ }^{7-11}$

While optically pumped NW lasers have been demonstrated, ${ }^{7,12-15}$ further reducing the threshold is critical for many applications as well as realizing electrically injected devices. ${ }^{16,17}$ Threshold reduction can be achieved by confining carriers within a nanostructure to benefit from the enhanced gain and improved temperature stability; ${ }^{18,19}$ both quantum dots (QDs) ${ }^{15}$ and coaxial quantum wells $(\mathrm{QWs})^{20}$ have been used as the gain medium. The use of coaxial QWs is particularly attractive, as it allows a much larger effective volume of gain material to be incorporated into the NWs, and the position of the QWs can be easily tailored to strongly overlap with the cavity mode.

There have been relatively few demonstrations of the use of coaxial QWs to reduce the threshold of NW lasers, ${ }^{21-23}$ with all reports using III-III-V, common group-V material systems (e.g., AlGaAs/GaAs), ${ }^{24-30}$ which have some disadvantages associated with the challenges of growing III-III-V core NWs. During the NW growth, the vast majority of the groupIII atoms diffuse from the substrate and NW sidewalls to the growth front. ${ }^{31}$ Different group-III elements have very different diffusion lengths, which are also highly temperature sensitive. Therefore, there are large differences in the optimal growth conditions for each species, especially in the growth temperature. ${ }^{32,33}$ This makes the growth of III-III-V NWs difficult, especially for the self-catalyzed growth mode. ${ }^{32,34}$ As a result, III-III-V NWs with QWs are commonly built on binary core
NWs. For example, AlGaAs/GaAs QWs use a GaAs core NW. Due to the lower band gap of bulk GaAs compared to AlGaAs and the GaAs QW, the core NW forms a lower potential region, which may trap a large number of carriers (Supporting Information Figure 1a). Strong core emission has been observed in this type of QWs, lowering the optical efficiency of the active region. ${ }^{35}$ Although GaAs NWs with InGaAs QWs do not have a lower band gap core, and lasing from this system has been reported, ${ }^{36}$ it appears to be difficult to obtain deep electrons and wells, even for high In compositions and hence significant strain. In contrast, group-V elements do not have the diffusion-length issue and III-V-V NWs can easily be grown with a large composition range. ${ }^{37}$ Therefore, III-V-V NWs with QWs can easily circumvent carrier trapping issues (Supporting Information Figure $1 \mathrm{~b}$ ). However, III-V-V-type NWs containing coaxial QWs have not been studied in any detail, and little is known about their basic structural, electronic, and optical properties. To the best of our knowledge, there have been no reports of lasers based on this type of structure.

Strong carrier confinement by deep QWs is expected to be very important given the high surface-to-volume ratio of the NWs and the close proximity of the active material to the surface, which can have a high density of carrier traps. ${ }^{38}$ Achieving deep confinement potentials for both electrons and holes requires semiconductor pairs with large band gap differences, but this can result in a high level of strain. The small NW size may allow the accommodation of high strain without the formation of dislocations, but there has been no study to date of highly strained NWs containing QWs and their application to lasers. A systematic and detailed study is thus needed, in particular of the relationship between the QW depth, carrier confinement, and lasing performance. If strained 

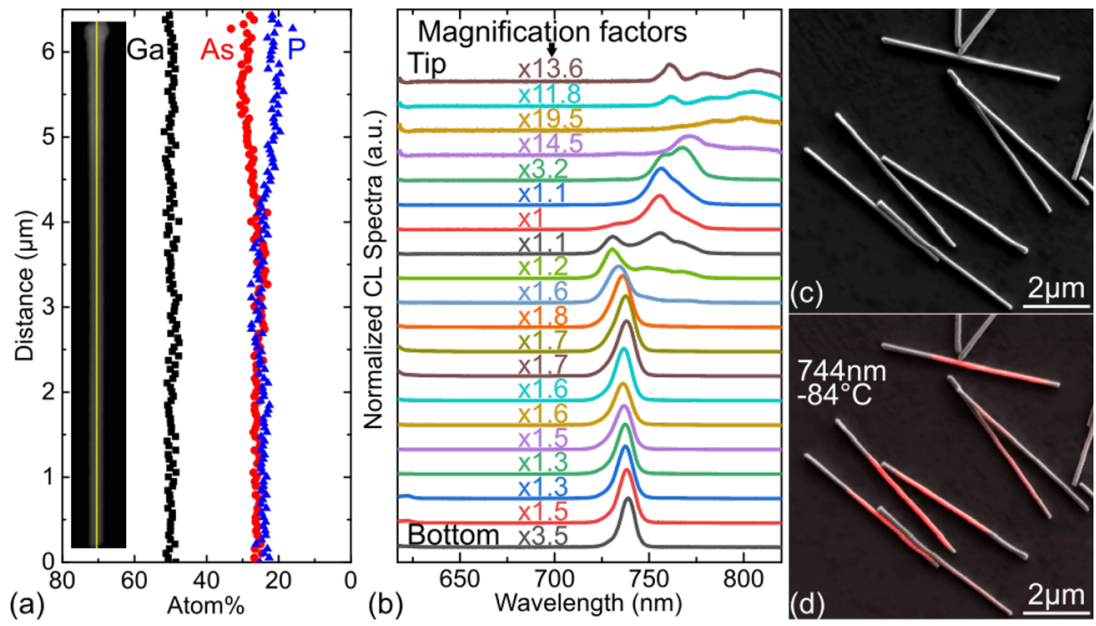

Figure 2. Structural and optical properties of the single QW NW. (a) EDX line profiles of Ga, As, and P compositions along the length of the NW, as shown in the inset. (b) Normalized cathodoluminescence $(\mathrm{CL})$ spectra recorded at $8 \mathrm{~K}$ along the NW length with constant spatial increments. (c) Scanning electron microscope image and (d) CL intensity mapping of the QW emission at $744 \mathrm{~nm}$.

deep QWs can demonstrate their suitability for NW lasers, this will greatly expand material choice, providing more freedom in device design.

The ternary III-V-V material, GaAsP, has a band gap that covers wavelengths ranging from green $(550 \mathrm{~nm})$ to nearinfrared $(860 \mathrm{~nm})$ at room temperature. ${ }^{39}$ Consequently, it is one of the most promising III-V compound semiconductors for visible emitters. In this paper, we report a detailed structural and optical study of strained GaAsP-based deep coaxial GaAs QWs and demonstrate their suitability for NW lasers.

\section{RESULTS AND DISCUSSION}

All NW samples were grown directly on p-type $\mathrm{Si}(111)$ substrates by solid-source III-V molecular beam epitaxy (MBE). NW growth was initiated via Ga droplets deposited on the $\mathrm{Si}$ substrate. Core-shell GaAsP NWs containing a single GaAs coaxial QW were grown for initial structural and optical studies. Scanning transmission electron microscope (STEM) images show that the first third of the NW length has a pure zinc-blende (ZB) structure without stacking faults, the middle third is lightly twinned, and the final third, toward the tip, is more defective (Figure 1a, Supporting Information S2). The diameter is relatively uniform but increases slightly toward the tip, where the presence of stacking faults creates new facets with higher surface energy, allowing faster shell growth. ${ }^{40}$ These stacking faults can be fully eliminated by our recently developed modified growth, which produces defect-free NWs. ${ }^{41}$ The $\mathrm{GaAs}_{0.62} \mathrm{P}_{0.38} \mathrm{NW}$ core has a diameter of $\sim 50$ $\mathrm{nm}$ (Figure $1 \mathrm{~b}$ ), and the GaAs $\mathrm{QW}$ is $3.5-10 \mathrm{~nm}$ thick, confined by $\sim 18 \mathrm{~nm} \mathrm{GaAs}{ }_{0.54} \mathrm{P}_{0.46}$ barriers. The $\mathrm{QW}$ width asymmetry is due to a shadowing effect during growth, caused by the random positioning of neighboring NWs. This nonuniformity can be seen in the emission spectra of some NWs, where multiple QW emission peaks are observed. This nonuniformity, which is likely to influence lasing performance, can be eliminated using patterned substrates to give uniformly spaced NWs. ${ }^{4}$ Both QW interfaces have a transition width of $\sim 1 \mathrm{~nm}$ (Figure 1c and d), which is much sharper than typical AlGaAs/GaAs NW interfaces. ${ }^{30,42}$ Despite a large compressive GaAs QW strain of $\sim 1.7 \%$, no strain-induced line- or loop-type dislocations are seen in the cross-section and side views
(Figure 1c and f). ${ }^{43,44}$ This absence of dislocations is further confirmed by the fast Fourier transform filtered images and strain mapping shown in the Supporting Information, Figures S3 and S4.

These studies demonstrate that it is possible to grow highly strained coaxial QWs with excellent crystalline quality. The ability to apply high levels of compressive strain to the GaAs QW should be advantageous for lasing action due to the increased heavy-hole-light-hole separation and the modification of the in-plane hole mass, which allows a given gain to be produced by a lower carrier density. ${ }^{45}$ Reduced threshold currents for compressively strained planar QW lasers have been demonstrated for a number of material systems, but require the use of an alloy QW to achieve the desired strain. ${ }^{46}$ The current NW system allows the application of large compressive strains to potentially higher quality binary GaAs QWs; this is not possible for planar growth on a GaAs substrate.

The initial $\sim 2 / 3$ of the NW has a uniform composition (Figure 2a), and this is reflected in the excellent spatial QW homogeneity (emission intensity, line width, and central wavelength), as demonstrated by the highly uniform cathodoluminescence (CL) emission (Figure 2b, c, and d). Within the first half of the NW the QW emission is constant to within $3 \mathrm{~nm}(\equiv 7 \mathrm{meV})$, followed by a small initial blue shift of the CL emission beyond the midpoint of the NW, suggesting a slight reduction of the QW width. No dark spots are visible in the CL image (Figure 2d), consistent with the absence of dislocations in the shell and QW. Comparison of CL and PL emission wavelengths with NextNano simulations ${ }^{47}$ suggests a QW width of $\sim 6.5-9 \mathrm{~nm}$ (Supporting Information S4), with the blue shift with respect to bulk GaAs arising from a combination of quantum confinement and the $1.7 \%$ compressive strain of the GaAs well. The energy separation between the lowest $\mathrm{QW}$ states and the barriers is calculated to be $\sim 115$ and $\sim 300 \mathrm{meV}$ for electrons and holes, respectively. The tip of the NW has a lower phosphorus composition (Figure $2 \mathrm{a}$ ) and emits at a longer wavelength of $>750 \mathrm{~nm}$, consistent with the previous observation (Figure 1a) that the defective tip region has a faster growth rate and hence a larger GaAs QW width. In addition, the emission at the tip is much 

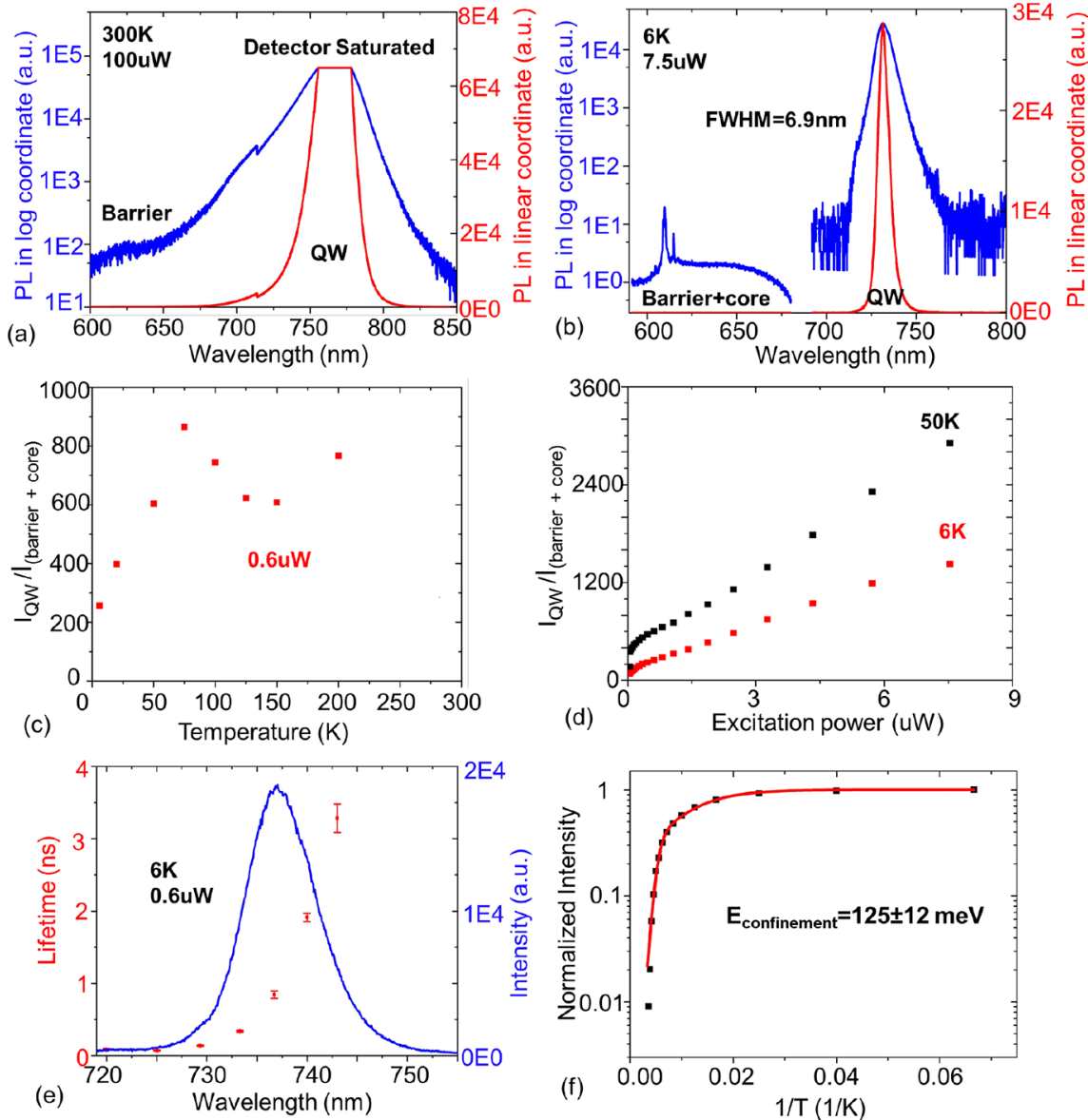

Figure 3. Carrier collection and confinement properties of the GaAs-GaAsP NW QW. (a) $300 \mathrm{~K}$ and (b) $6 \mathrm{~K}$ micro-photoluminescence ( $\mu$ PL) spectra from a stacking-fault-free segment of a single NW. The discontinuity in (a) at $\sim 720 \mathrm{~nm}$ results from the stitching together of two spectra. (c) Temperature-dependent and (d) power-dependent ratio of the integrated QW emission (691-808 $\mathrm{nm}$ ) to the barrier and core emission (591-680 nm). (e) PL spectrum (blue) and carrier lifetimes (red) measured from a stacking-fault-free segment of the NW. (f) Integrated and normalized ensemble PL intensity as a function of inverse temperature.

weaker, with the broad emission extending below the bulk GaAs band gap, in accordance with the presence of defects. ${ }^{48}$

Micro-photoluminescence ( $\mu$-PL) spectra are excited by a $515 \mathrm{~nm}$ diode laser which is absorbed by the QW and also both the core and barrier GaAsP. The NWs are mechanically transferred to a new Si wafer and are studied normal to their length. The $300 \mathrm{~K}$ spectra of a single NW (Figure 3a) are dominated by the QW emission, with the barrier emission only weakly visible. No NW core emission is observed, despite this region forming a potential carrier reservoir due to a lower $\mathrm{P}$ composition and constituting $\sim 20 \%$ of the total NW volume (Figure $1 \mathrm{~b}$ and $\mathrm{c}$ ). This indicates very efficient carrier transfer from the GaAsP regions to the QW. At $6 \mathrm{~K}$ the photoexcited carriers are much less mobile, ${ }^{49}$ and a very broad but weak emission is observed in the range 590-680 nm (Figure 3b) due to the QW barriers and NW core. The QW emission has a narrow full width at half maximum (fwhm) of $6.9 \mathrm{~nm}$ (三16 $\mathrm{meV}$ ) and is $\sim 10^{3}$ more intense than that of the GaAsP. The ratio of the integrated $\mathrm{QW}$ emission $(691-808 \mathrm{~nm})$ to that of the barrier and core emission (591-680 nm) exceeds 200 over the temperature range $6-300 \mathrm{~K}$ for low excitation power (Figure 3c); this ratio increases rapidly with excitation power (Figure $3 \mathrm{~d}$ ). The lifetime of the $\mathrm{QW}$-confined carriers increases with increasing wavelength (decreasing energy) (Figure 3e, Supporting Information S5), reflecting state filling and the transfer of carriers from high to low energy states within the QW. The carrier lifetime at the emission peak is $\sim 1$ ns. An analysis of the temperature dependence of the QW PL intensity, measured from an ensemble of NWs (Figure 3f), indicates a large barrier of $125 \pm 12 \mathrm{meV}$ against carrier loss (Supporting Information S6), which is consistent with the calculated electron localization energy of $115 \mathrm{meV}$ discussed above. It is also found that carrier loss due to surface nonradiative recombination is greatly reduced in the present structure (Supporting Information S6).

Our results demonstrate that the deep coaxial QWs act to efficiently capture photoexcited carriers, because the NW diameter $(<200 \mathrm{~nm})$ is much smaller than the carrier diffusion length (in the micrometer range). ${ }^{50,51}$ Captured carriers are strongly confined by the deep QWs, resulting in extremely weak barrier emission at all temperatures. In addition, the strong confinement inhibits carrier loss to surface states (Supporting Information S6). As a result, the QW NW has a much stronger PL emission $(\sim 150 \times)$ compared to NWs with comparable morphology and density but without a QW (Supporting Information S7 and Figure S8).

NWs with three coaxial QWs were grown in order to provide the gain required for lasing. The average composition of the NW core is $\mathrm{GaAs}_{0.38} \mathrm{P}_{0.62}$, and the barriers are $\mathrm{GaAs}_{0.53} \mathrm{P}_{0.47}$ (Figure $4 \mathrm{a}$ ). The diameter of the NWs is $\sim 400$ $\mathrm{nm}$. Finite-difference time-domain (FDTD) simulations shown in Figure $4 \mathrm{~b}$ show incomplete overlap between the QWs and 

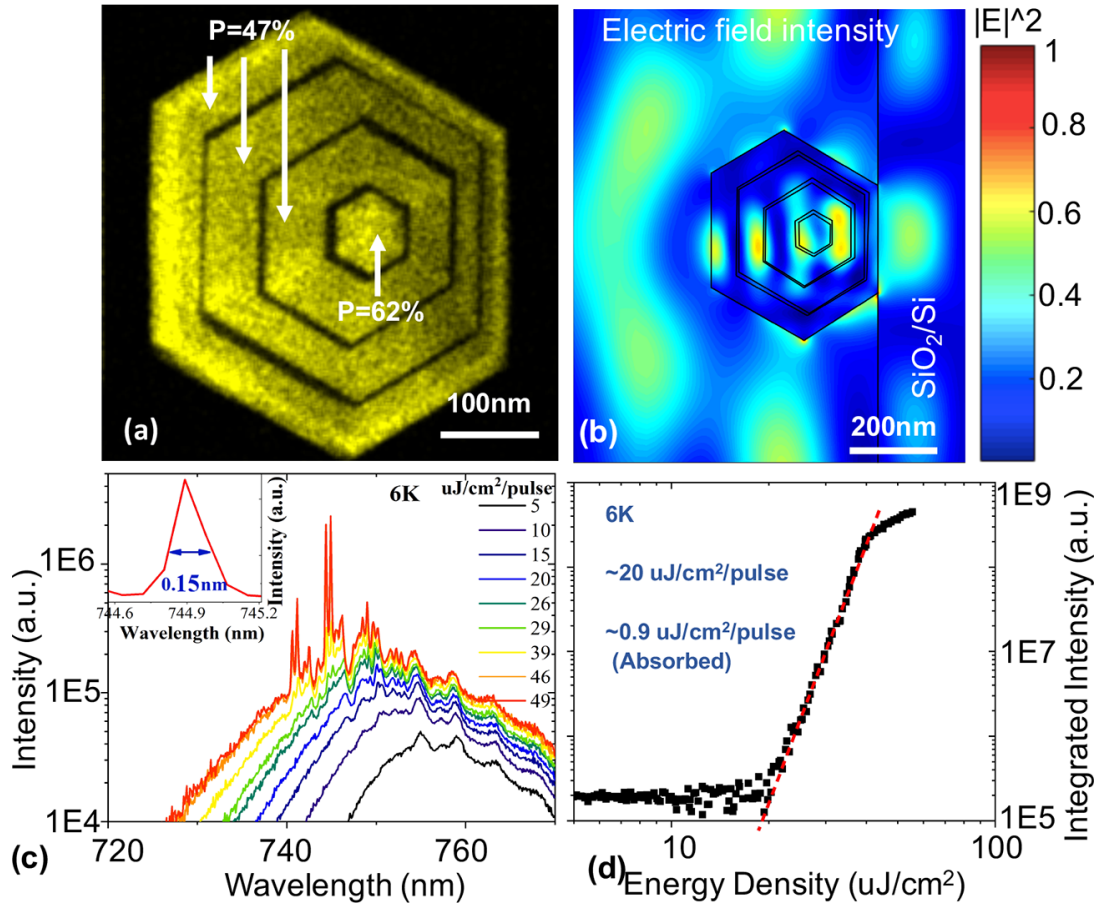

Figure 4. Structural and lasing characteristics of a single NW with three coaxial QWs at $6 \mathrm{~K}$. (a) EDX mapping of the phosphorus content across the NW clearly showing the three QWs. (b) FDTD-simulated electric field intensity distribution in the cross-section of the nanowire when illuminated with a Gaussian beam $(\lambda=635 \mathrm{~nm})$. (c) Pulsed-excited emission spectra for a sample temperature of $6 \mathrm{~K}$. The inset shows a lasing mode for excitation of $49 \mu \mathrm{J} / \mathrm{cm}^{2} /$ pulse. (d) Double-logarithmic integrated output-power intensity of the lasing mode versus incident pump pulse energy density.

cavity mode. A pulsed laser, with a focused elliptical spot of 30 $\times 1.4 \mu \mathrm{m}^{2}$, was used to excite the $\mathrm{NW}$ at $6 \mathrm{~K}$. The excitation wavelength of $635 \mathrm{~nm}$ is only absorbed by the QW and not the NW core or barriers. With increasing pulse energy, the broad spontaneous emission shifts toward higher energy due to QW state filling (Figure 4c). Fabry-Pérot cavity modes at almost constant wavelengths are observed, superimposed on the spontaneous emission. At high pump energies, a small number of modes increase superlinearly with energy, consistent with the onset of lasing (Figure $4 \mathrm{~d}$ ) at a threshold of $\sim 20 \mu \mathrm{J} / \mathrm{cm}^{2}$ / pulse. FDTD simulations indicate that only $4.4 \%$ of the incident laser power is absorbed by the NW, which further reduces the absorbed threshold flux to $\sim 0.9 \mu \mathrm{J} / \mathrm{cm}^{2} /$ pulse.

The inset to Figure $4 \mathrm{c}$ shows the dominant laser mode, which has a low line width of $\sim 0.15 \mathrm{~nm}(\equiv 0.34 \mathrm{meV})$. This is narrower than the majority of previously reported values for III-V NW lasers (typically $>0.7 \mathrm{~nm})^{7,14,15,20,52-57}$ and comparable to the lowest reported (resolution limited) value of $\sim 0.25 \mathrm{meV}$. $^{58}$ This suggests relatively low levels of chirp in the current laser.

Three approaches should further improve the performance of the current coaxial QW lasers. The first is eliminating the defects, which form at the tip of the NW and which may consume carriers by nonradiative recombination, particularly at high temperatures. This should improve the temperature performance, which, for the current structure, limits lasing to below $\sim 100 \mathrm{~K}$ under our measurement conditions. Second, due to unoptimized droplet consumption, the top facet has a low quality (Figure 1a and inset of Figure 2a). Improving this will provide a higher quality optical cavity. Third, improving the QW width homogeneity by growing the NWs on prepatterned substrates will narrow the spectral distribution of the gain, allowing the threshold gain to be reached for lower carrier injection.

\section{CONCLUSIONS}

In summary, the properties of deep and highly strained GaAsP/GaAs coaxial NW QWs have been studied. The QW is highly uniform along the NW length for the region where the NW is defect free. Despite the large compressive strain, no strain-induced dislocations are observed in the QW region. The QW exhibits very efficient carrier collection and strong carrier confinement, resulting in the PL being dominated by the QW emission at all temperatures. In addition, carrier localization in the QW appears to reduce the influence of surface states, further contributing to the high radiative efficiency. A NW laser structure containing three coaxial QWs demonstrates a very low external (internal) threshold of $20(0.9) \mu \mathrm{J} / \mathrm{cm}^{2} /$ pulse and a very small lasing line width of $\sim 0.15 \mathrm{~nm}$. The current system offers a number of significant advantages compared to previously studied systems, including deep electron and hole wells, a high compressively strained binary QW, and the absence of a low band gap core, which can act as a carrier sink. These initial results demonstrate that highly strained III-V-V coaxial QWs are suitable for the fabrication of low-threshold NW lasers, with a number of advantages compared to more commonly studied systems.

\section{EXPERIMENTAL METHODS}

NW Growth. The self-catalyzed GaAsP NWs were grown directly on p-type $\mathrm{Si}(111)$ substrates by solid-source III-V MBE. ${ }^{59}$ For the single QW NWs, the core $\mathrm{GaAs}_{0.62} \mathrm{P}_{0.38} \mathrm{NW}$ was grown with a $\mathrm{Ga}$ beam equivalent pressure, $\mathrm{V} / \mathrm{III}$ flux ratio, $\mathrm{P} /(\mathrm{As}+\mathrm{P})$ flux ratio, substrate temperature, and growth duration of $8.41 \times 10^{-8}$ Torr, $\sim 40$, $25 \%, \sim 640{ }^{\circ} \mathrm{C}$, and $1.5 \mathrm{~h}$, respectively. After the growth of the core, 
the Ga droplets were consumed by closing the Ga flux and keeping the As and $\mathrm{P}$ fluxes open. Two $\mathrm{GaAs}_{0.54} \mathrm{P}_{0.46}$ shells were then grown with a Ga beam equivalent pressure, $\mathrm{V} / \mathrm{III}$ flux ratio, $\mathrm{P} /(\mathrm{As}+\mathrm{P})$ flux ratio, substrate temperature, and growth duration of $8.41 \times 10^{-8}$ Torr, $50,50 \%, \sim 550{ }^{\circ} \mathrm{C}$, and $30 \mathrm{~min}$, respectively. A $\sim 10 \mathrm{~nm}$ GaAs QW was grown between the two GaAsP shells, with a Ga beam equivalent pressure, $\mathrm{V} / \mathrm{III}$ flux ratio, substrate temperature, and growth duration of $8.41 \times 10^{-8}$ Torr, $60, \sim 550{ }^{\circ} \mathrm{C}$, and $10 \mathrm{~min}$, respectively. For the three-QW sample, the core $\mathrm{GaAs}_{0.38} \mathrm{P}_{0.62} \mathrm{NW}$ was grown with a $\mathrm{Ga}$ beam equivalent pressure, $\mathrm{V} / \mathrm{III}$ flux ratio, $\mathrm{P} /(\mathrm{As}+\mathrm{P})$ flux ratio, substrate temperature, and growth duration of $8.41 \times 10^{-8}$ Torr, $\sim 30$, $41 \%, \sim 640{ }^{\circ} \mathrm{C}$, and $1.5 \mathrm{~h}$, respectively. After the droplet consumption, the three GaAs QWs and three $\mathrm{GaAs}_{0.53} \mathrm{P}_{0.47}$ barriers were grown with same condition as that of the single $\mathrm{QW}$ sample, except that the growth time was $1 \mathrm{~h}$ for each barrier. Finally, shells of $\mathrm{Al}_{0.5} \mathrm{Ga}_{0.5} \mathrm{As}_{0.53} \mathrm{P}_{0.47}(30 \mathrm{~min})$ and $\mathrm{GaAs}_{0.53} \mathrm{P}_{0.47}(20 \mathrm{~min})$ were deposited. The final NWs have a diameter of $\sim 400 \mathrm{~nm}$ and an asgrown length of $9.4 \mu \mathrm{m}$. During growth, the substrate temperature was measured by a pyrometer.

Transmission Electron Microscopy (TEM). Simple mechanical transfer of the NWs onto a lacey carbon support was used to prepare TEM specimens. The cross-section samples were prepared by embedding the NWs in a low-viscosity resin and slicing using a microtome. The TEM measurements were performed with JEOL 2100 and doubly corrected ARM200F microscopes, both operating at $200 \mathrm{kV}$.

Cathodoluminescence. Imaging was performed on both NWs still attached to the substrates and also broken off and transferred to a $\mathrm{Si}$ substrate. The studies were carried out in a dedicated SEM at a temperature of $8 \mathrm{~K}$, using an acceleration voltage of $5 \mathrm{kV}$ and a probe current of $25 \mathrm{pA}$. The detector was a Si-CCD. CL measurements shown in Figure $2 c$ were performed using a Gatan MonoCL3 system within a Zeiss Supra 55VP SEM operating at $3 \mathrm{kV}$.

Photoluminescence. Large-area PL measurements (Figure 2b) probe a high number of NWs and is relatively insensitive to movement of the laser spot, which tends to occur as the temperature is varied. Excitation is by a $532 \mathrm{~nm}$ diode-pumped solid-state laser, with a $0.25 \mathrm{~m}$ monochromator used to disperse the $\mathrm{PL}$, which is detected by a TE-cooled Si detector. $\mu$-PL spectra were obtained from single NWs, which had been removed from the original substrate and transferred to a new Si wafer. $\mu$-PL spectra of single NWs were excited by a $\mathrm{cw} 515 \mathrm{~nm}$ diode laser. The time-resolved PL of the single QW sample and the pumping of the MQW laser sample was performed using a pulsed $635 \mathrm{~nm}$ diode laser with a repetition frequency of 80 $\mathrm{MHz}$ and $80 \mathrm{ps}$ pulse width. The samples were measured under vacuum inside a continuous flow cryostat (base temperature $6 \mathrm{~K}$ ). The incident laser was focused with a $20 \times$ long working distance microscope objective to a spot size of $\sim 1 \mu \mathrm{m}$ diameter. The resultant $\mathrm{PL}$ was collected by the same microscope objective and focused into a $0.75 \mathrm{~m}$ spectrometer, where the spectral components were resolved and detected using a $300 \mathrm{l} / \mathrm{mm}$ grating and a nitrogen-cooled CCD. The spectral resolution was $\sim 0.5 \mathrm{meV}$, and higher resolution measurements were recorded using an 1800 lines $/ \mathrm{mm}$ grating with a resolution of $0.09 \mathrm{meV}$. The time-resolved PL was detected by a silicon APD and a photon counting card. The resolution of this system was $\sim 100$ ps.

FDTD Simulations. FDTD simulations were performed using Lumerical FDTD Solutions, with a custom source (free-space wavelength of $635 \mathrm{~nm}$, elliptical Gaussian beam with an fwhm of 1.3 and $30 \mu \mathrm{m}$ along orthogonal directions). The source was injected perpendicular to the nanowire and substrate $\left(200 \mathrm{~nm} \mathrm{SiO}{ }_{2}\right.$ covered $\mathrm{Si}$ ). The nanowire structure was modeled from the cross-sectional TEM image, with a complex refractive index value for the GaAs QW $(3.85+0.192 \mathrm{i})$ and no absorption for the $\mathrm{Ga}_{x} \mathrm{AsP}_{1-x}$ barriers $(3.6+$ $0 \mathrm{i})$. A fine mesh ( $2 \mathrm{~nm}$ resolution) was used to resolve the multiple QW heterostructure. Field monitors were used to record the field in the nanowire cross-section, and the fraction of pump power absorbed was calculated from the net power transmission through a box of monitors surrounding the nanowire.

\section{ASSOCIATED CONTENT}

\section{Supporting Information}

The Supporting Information is available free of charge on the ACS Publications website at DOI: 10.1021/acsnano.9b01775.

Schematic of AlGaAs/GaAs and GaAsP/GaAs nanowire quantum wells; crystal information on single quantum well NWs; dislocation analysis of the GaAsP-GaAs interfaces and strain distribution in the multiple QWs; calculation of QW electron and hole confinement energies; lifetime dynamics of the QW carriers; temperature dependence of the photoluminescence; significantly enhanced PL emission via the addition of a QW (PDF)

\section{AUTHOR INFORMATION}

\section{Corresponding Author}

*E-mail: yunyang.zhang.11@ucl.ac.uk.

ORCID

Yunyan Zhang: 0000-0002-2196-7291

H. Aruni Fonseka: 0000-0003-3410-6981

Anders Gustafsson: 0000-0001-9289-5961

Patrick W. Parkinson: 0000-0001-9429-9768

Ana M. Sanchez: 0000-0002-8230-6059

David J. Mowbray: 0000-0002-7673-6837

Notes

The authors declare no competing financial interest.

\section{ACKNOWLEDGMENTS}

The authors acknowledge the support of Leverhulme Trust, EPSRC grants EP/P000886/1, EP/P006973/1, EP/P000916/ 1, and EPSRC National Epitaxy Facility. J.A.G. was supported by EPSRC EP/N509796/1. A.G. acknowledges support from the Swedish Research Counsel.

\section{REFERENCES}

(1) Cui, Y.; Wei, Q. Q.; Park, H. K.; Lieber, C. M. Nanowire Nanosensors for Highly Sensitive and Selective Detection of Biological and Chemical Species. Science 2001, 293, 1289-1292.

(2) Wang, J.; Gudiksen, M. S.; Duan, X.; Cui, Y.; Lieber, C. M. Highly Polarized Photoluminescence and Photodetection from Single Indium Phosphide Nanowires. Science 2001, 293, 1455-1457.

(3) Yan, R.; Gargas, D.; Yang, P. Nanowire photonics. Nat. Photonics 2009, 3, 569-576.

(4) Li, K. H.; Liu, X.; Wang, Q.; Zhao, S.; Mi, Z. Ultralow-Threshold Electrically Injected AlGaN Nanowire Ultraviolet Lasers on $\mathrm{Si}$ Operating at Low Temperature. Nat. Nanotechnol. 2015, 10, 140144.

(5) Zhang, Y.; Wu, J.; Aagesen, M.; Holm, J.; Hatch, S.; Tang, M.; Huo, S.; Liu, H. Self-Catalyzed Ternary Core-Shell GaAsP Nanowire Arrays Grown on Patterned Si Substrates by Molecular Beam Epitaxy. Nano Lett. 2014, 14, 4542-4547.

(6) Frost, T.; Jahangir, S.; Stark, E.; Deshpande, S.; Hazari, A.; Zhao, C.; Ooi, B. S.; Bhattacharya, P. Monolithic Electrically Injected Nanowire Array Edge-Emitting Laser on (001) Silicon. Nano Lett. 2014, 14, 4535-4541.

(7) Saxena, D.; Mokkapati, S.; Parkinson, P.; Jiang, N.; Gao, Q.; Tan, H. H.; Jagadish, C. Optically Pumped Room-Temperature GaAs Nanowire Lasers. Nat. Photonics 2013, 7, 963-988.

(8) Fischer, R.; Masselink, W. T.; Klem, J.; Henderson, T.; McGlinn, T. C.; Klein, M. V.; Morkoç, H.; Mazur, J. H.; Washburn, J. Growth and Properties of GaAs/AlGaAs on Nonpolar Substrates Using Molecular Beam Epitaxy. J. Appl. Phys. 1985, 58, 374-381. 
(9) Kim, H.; Lee, W. J.; Farrell, A. C.; Morales, J. S.; Senanayake, P.; Prikhodko, S. V.; Ochalski, T. J.; Huffaker, D. L. Monolithic InGaAs Nanowire Array Lasers on Silicon-on-Insulator Operating at Room Temperature. Nano Lett. 2017, 17, 3465-3470.

(10) Liang, D.; Bowers, J. E. Recent Progress in Lasers on Silicon. Nat. Photonics 2010, 4, 511-517.

(11) Won, R.; Mario, P. Integrating Silicon Photonics. Nat. Photonics 2010, 4, 498-499.

(12) Guilhabert, B.; Hurtado, A.; Jevtics, D.; Gao, Q.; Tan, H. H.; Jagadish, C.; Dawson, M. D. Transfer Printing of Semiconductor Nanowires with Lasing Emission for Controllable Nanophotonic Device Fabrication. ACS Nano 2016, 10, 3951-3958.

(13) Xu, W. Z.; Ren, F. F.; Jevtics, D.; Hurtado, A.; Li, L.; Gao, Q.; Ye, J.; Wang, F.; Guilhabert, B.; Fu, L.; Lu, H.; Zhang, R.; Tan, H. H.; Dawson, M. D.; Jagadish, C. Vertically Emitting Indium Phosphide Nanowire Lasers. Nano Lett. 2018, 18, 3414-3420.

(14) Stettner, T.; Zimmermann, P.; Loitsch, B.; Döblinger, M.; Regler, A.; Mayer, B.; Winnerl, J.; Matich, S.; Riedl, H.; Kaniber, M.; Abstreiter, G.; Koblmüller, G.; Finley, J. J. Coaxial GaAs-AlGaAs Core-Multishell Nanowire Lasers with Epitaxial Gain Control. Appl. Phys. Lett. 2016, 108, 011108-1-011108-5.

(15) Tatebayashi, J.; Kako, S.; Ho, J.; Ota, Y.; Iwamoto, S.; Arakawa, Y. Room-Temperature Lasing In a Single Nanowire with Quantum Dots. Nat. Photonics 2015, 9, 501-505.

(16) Zhao, S.; Liu, X.; Woo, S. Y.; Kang, J.; Botton, G. A.; Mi, Z. An Electrically Injected AlGaN Nanowire Laser Operating in theUltraviolet-C Band. Appl. Phys. Lett. 2015, 107, 043101-1-043101-5.

(17) Duan, X.; Huang, Y.; Agarwal, R.; Lieber, C. M. SingleNanowire Electrically Driven Lasers. Nature 2003, 421, 241-245.

(18) Bhattacharya, P.; Mi, Z. Quantum-Dot OptoelectronicDevices. Proc. IEEE 2007, 95, 1723-1740.

(19) Nguyen, H. P. T.; Cui, K.; Zhang, S.; Djavid, M.; Korinek, A.; Botton, G. A.; Mi, Z. Controlling Electron Overflow In PhosphorFree InGaN/GaNNanowire White Light-Emitting Diodes. Nano Lett. 2012, 12, 1317-1323.

(20) Saxena, D.; Jiang, N.; Yuan, X.; Mokkapati, S.; Guo, Y.; Tan, H. H.; Jagadish, C. Design and Room-TemperatureOperation of GaAs/ AlGaAs Multiple Quantum WellNanowire Lasers. Nano Lett. 2016, $16,5080-5086$.

(21) Alanis, J. A.; Saxena, D.; Mokkapati, S.; Jiang, N.; Peng, K.; Tang, X.; Fu, L.; Tan, H. H.; Jagadish, C.; Parkinson, P. LargeScaleStatistics For ThresholdOptimization of OpticallyPumpedNanowire Lasers. Nano Lett. 2017, 17, 4860-4865.

(22) Qian, F.; Li, Y.; Gradečak, S.; Park, H. G.; Dong, Y.; Ding, Y.; Wang, Z. L.; Lieber, C. M. Multi-Quantum-WellNanowireHeterostructures for Wavelength-Controlled Lasers. Nat. Mater. 2008, 7, 701-706.

(23) Schuster, F.; Kapraun, J.; Malheiros-Silveira, G. N.; Deshpande, S.; Chang-Hasnain, C. J. Site-ControlledGrowth of MonolithicInGaAs/InP Quantum WellNanopillar Lasers on Silicon. Nano Lett. 2017, 17, 2697-2702.

(24) Park, K. W.; Park, C. Y.; Ravindran, S.; Jang, J. S.; Jo, Y. R.; Kim, B. J.; Lee, Y. T. Observation and Tunability of Room Temperature Photoluminescence of GaAs/GaInAsCore-MultipleQuantum-Well Shell Nanowire Structure Grown on Si (100) by MolecularBeamEpitaxy. Nanoscale Res. Lett. 2014, 9, 626-636.

(25) Fonseka, H. A.; Ameruddin, A. S.; Caroff, P.; Tedeschi, D.; De Luca, M.; Mura, F.; Guo, Y.; Lysevych, M.; Wang, F.; Tan, H. H.; Polimeni, A.; Jagadish, C. InP- $\operatorname{In}_{\mathrm{x}} \mathrm{Ga}_{1-\mathrm{x}}$ As Core-Multi-Shell Nanowire Quantum Wells withTunable Emission in the $1.3-1.55 \mu \mathrm{m}$ Wavelength Range. Nanoscale 2017, 9, 13554-13562.

(26) Yuan, X.; Saxena, D.; Caroff, P.; Wang, F.; Lockrey, M.; Mokkapati, S.; Tan, H. H.; Jagadish, C. StrongAmplifiedSpontaneous Emission from High Quality $\mathrm{GaAs}_{1-\mathrm{x}} \mathrm{Sb}_{\mathrm{x}}$ Single Quantum WellNanowires. J. Phys. Chem. C 2017, 121, 8636-8644.

(27) Dimakis, E.; Jahn, U.; Ramsteiner, M.; Tahraoui, A.; Grandal, J.; Kong, X.; Marquardt, O.; Trampert, A.; Riechert, H.; Geelhaar, L. Coaxial Multishell (In, Ga) As/GaAsNanowires for Near-Infrared Emission on Si Substrates. Nano Lett. 2014, 14, 2604-2609.
(28) Moewe, M.; Chuang, L. C.; Crankshaw, S.; Ng, K. W.; ChangHasnain, C. Core-Shell InGaAs/GaAs Quantum WellNanoneedlesGrown on SiliconwithSilicon-Transparent Emission. Opt. Express 2009, 17, 7831-7836.

(29) Yan, X.; Zhang, X.; Li, J.; Wu, Y.; Cui, J.; Ren, X. Fabrication and Optical Properties of GaAs/InGaAs/GaAsNanowireCore-Multishell Quantum WellHeterostructures. Nanoscale 2015, 7, 1110-1115.

(30) Fickenscher, M.; Shi, T.; Jackson, H. E.; Smith, L. M.; YarrisonRice, J. M.; Zheng, C.; Miller, P.; Etheridge, J.; Wong, B. M.; Gao, Q.; Deshpande, S.; Tan, H. H.; Jagadish, C. Optical, Structural, and Numerical Investigations of GaAs/AlGaAsCore-MultishellNanowire Quantum Well Tubes. Nano Lett. 2013, 13, 1016-1022.

(31) Colombo, C.; Spirkoska, D.; Frimmer, M.; Abstreiter, G.; FontcubertaiMorral, A. Ga-Assisted Catalyst-Free Growth Mechanism of GaAs Nanowires by Molecular Beam Epitaxy. Phys. Rev. B: Condens. Matter Mater. Phys. 2008, 77, 155326-1-155326-5.

(32) Paek, J.; Yamaguchi, M.; Amano, H. MBE-VLS Growth of Catalyst-Free III-V Axial Heterostructure Nanowires on $\left(\begin{array}{lll}1 & 1 & 1\end{array}\right) \mathrm{Si}$ Substrates. J. Cryst. Growth 2011, 323, 315-318.

(33) Kim, Y.; Joyce, H. J.; Gao, Q.; Tan, H. H.; Jagadish, C.; Paladugu, M.; Zou, J.; Suvorova, A. A. Influence of Nanowire Density on the Shape and Optical Properties of Ternary InGaAs Nanowires. Nano Lett. 2006, 6, 599-604.

(34) Heiss, M.; Ketterer, B.; Uccelli, E.; Morante, J. R.; Arbiol, J.; Imorral, A. F. In(Ga)As Quantum Dot Formation on Group-III Assisted Catalyst-Free InGaAs Nanowires. Nanotechnology 2011, 22, 195601-195608.

(35) Shi, T.; Jackson, H. E.; Smith, L. M.; Jiang, N.; Gao, Q.; Tan, H. H.; Jagadish, C.; Zheng, C.; Etheridge, J. Emergence of Localized States in Narrow GaAs/AlGaAs Nanowire Quantum Well Tubes. Nano Lett. 2015, 15, 1876-1882.

(36) Stettner, T.; Thurn, A.; Döblinger, M.; Hill, M. O.; Bissinger, J.; Schmiedeke, P.; Matich, S.; Kostenbader, T.; Ruhstorfer, D.; Riedl, H.; Kaniber, M.; Lauhon, L. J.; Finley, J. J.; Koblmüller, G. Tuning Lasing Emission Toward Long Wavelengths in GaAs-(In, Al) GaAs Core-Multishell Nanowires. Nano Lett. 2018, 18, 6292-6300.

(37) Zhang, Y.; Aagesen, M.; Holm, J. V.; Jørgensen, H. I.; Wu, J.; Liu, H. Self-Catalyzed GaAsP Nanowires Grown on Silicon Substrates by Solid-Source Molecular Beam Epitaxy. Nano Lett. 2013, 13, 38973902.

(38) Joyce, H. J.; Docherty, C. J.; Gao, Q.; Tan, H. H.; Jagadish, C.; Lloyd-Hughes, J.; Herz, L. M.; Johnston, M. B. Electronic Properties of GaAs, InAs and InP Nanowires Studied by Terahertz Spectroscopy. Nanotechnology 2013, 24, 214006-214013.

(39) Vurgaftman, I.; Meyer, J. R.; Ram-Mohan, L. R. Band Parameters for III-V Compound Semiconductors and Their Alloys. J. Appl. Phys. 2001, 89, 5815-5875.

(40) Zou, J.; Paladugu, M.; Wang, H.; Auchterlonie, G. J.; Guo, Y. N.; Kim, Y.; Gao, Q.; Joyce, H. J.; Tan, H. H.; Jagadish, C. Growth Mechanism of Truncated Triangular III-V Nanowires. Small 2007, 3, 389-393.

(41) Zhang, Y.; Fonseka, H. A.; Aagesen, M.; Gott, J. A.; Sanchez, A. M.; Wu, J.; Jurczak, P.; Huo, S.; Liu, H. Growth of Pure Zinc-Blende GaAs(P) Core-Shell Nanowires with Highly Regular Morphology. Nano Lett. 2017, 17, 4946-4950.

(42) Badada, B. H.; Shi, T.; Jackson, H. E.; Smith, L. M.; Zheng, C.; Etheridge, J.; Gao, Q.; Tan, H. H.; Jagadish, C. Quantum Confined Stark Effect in a GaAs/AlGaAs Nanowire Quantum Well Tube Device: Probing Exciton Localization. Nano Lett. 2015, 15, 78477852.

(43) Popovitz-Biro, R.; Kretinin, A.; Von Huth, P.; Shtrikman, H. InAs/GaAs Core-Shell Nanowires. Cryst. Growth Des. 2011, 11, $3858-3865$

(44) Kavanagh, K. L. Misfit Dislocations in Nanowire Heterostructures. Semicond. Sci. Technol. 2010, 25, 024006-024013.

(45) Gain and recombination current in quantum wells. In Quantum Confined Laser Devices: Optical Gain and Recombination in Semiconductors; Blood, P., Ed.; OUP Oxford: Oxford, 2015; pp 205-206. 
(46) Thiis, P. J. A.; Tiemeijer, L. F.; Binsma, J. J. M.; Van Dongen, T. Progress in Long-Wavelength Strained-Layer InGaAs(P) Quantum-Well Semiconductor Lasers and Amplifiers. IEEE J. Quantum Electron. 1994, 30, 477-499.

(47) Birner, S.; Zibold, T.; Lauer, T.; Kubis, T.; Sabathil, M.; Trellakis, A.; Vogl, P. Nextnano: General Purpose 3-D Simulations. IEEE Trans. Electron Devices 2007, 54, 2137-2142.

(48) Sanchez, A. M.; Gott, J. A.; Fonseka, H. A.; Zhang, Y.; Liu, H.; Beanland, R. Stable Defects in Semiconductor Nanowires. Nano Lett. 2018, 18, 3081-3087.

(49) Stillman, G. E.; Wolfe, C. M.; Dimmock, J. O. Hall Coefficient Factor for Polar Mode Scattering in n-Type GaAs. J. Phys. Chem. Solids 1970, 31, 1199-1204.

(50) http://www.ioffe.ru/SVA/NSM/Semicond/GaP/electric. html\#Recombination (accessed May 4, 2019).

(51) http://www.ioffe.ru/SVA/NSM/Semicond/GaAs/electric. html\#Recombination (accessed May 4, 2019).

(52) Ren, D.; Ahtapodov, L.; Nilsen, J. S.; Yang, J.; Gustafsson, A.; Huh, J.; Conibeer, G. J.; Van Helvoort, A. T.; Fimland, B. O.; Weman, H. Single-Mode Near-Infrared Lasing in a GaAsSb-Based Nanowire Superlattice at Room Temperature. Nano Lett. 2018, 18, 2304-2310.

(53) Ho, J.; Tatebayashi, J.; Sergent, S.; Fong, C. F.; Ota, Y.; Iwamoto, S.; Arakawa, Y. A Nanowire-Based Plasmonic Quantum Dot Laser. Nano Lett. 2016, 16, 2845-2850.

(54) Wei, W.; Liu, Y.; Zhang, X.; Wang, Z.; Ren, X. EvanescentWave Pumped Room-Temperature Single-Mode GaAs/AlGaAs CoreShell Nanowire Lasers. Appl. Phys. Lett. 2014, 104, 223103-1223103-4.

(55) Hua, B.; Motohisa, J.; Kobayashi, Y.; Hara, S.; Fukui, T. Single GaAs/GaAsP Coaxial Core-Shell Nanowire Lasers. Nano Lett. 2009, 9, 112-116.

(56) Chen, S.; Jansson, M.; Stehr, J. E.; Huang, Y.; Ishikawa, F.; Chen, W. M.; Buyanova, I. A. Dilute Nitride Nanowire Lasers Based on a GaAs/GaNAs Core/Shell Structure. Nano Lett. 2017, 17, 17751781.

(57) Johnson, J. C.; Choi, H. J.; Knutsen, K. P.; Schaller, R. D.; Yang, P.; Saykally, R. J. Single Gallium Nitride Nanowire Lasers. Nat. Mater. 2002, 1, 106-110.

(58) Mayer, B.; Rudolph, D.; Schnell, J.; Morkötter, S.; Winnerl, J.; Treu, J.; Müller, K.; Bracher, G.; Abstreiter, G.; Koblmüller, G.; Finley, J. J. Lasing from Individual GaAs-AlGaAs Core-Shell Nanowires up to Room Temperature. Nat. Commun. 2013, 4, 2931-2938.

(59) Zhang, Y.; Sanchez, A. M.; Sun, Y.; Wu, J.; Aagesen, M.; Huo, S.; Liu, H. Influence of Droplet Size on the Growth of Self-Catalyzed Ternary GaAsP Nanowires. Nano Lett. 2016, 16, 1237-1243. 